\title{
RESEARCH
}

\section{SRC1 deficiency in hypothalamic arcuate nucleus increases appetite and body weight}

\author{
Qianqian Lu¹, Yuying Yang², Sheng Jia², Shaoqiang Zhao², Bin Gu², Peng Lu¹, Yang He², Ruixin Liu², Jiqiu Wang², \\ Guang Ning1,2 and Qinyun Ma²
}

1The Institute of Health Sciences, Shanghai Institutes for Biological Sciences, University of Chinese Academy of Sciences, Chinese Academy of Sciences, Shanghai, China

2Department of Endocrinology and Metabolism, Shanghai Institute of Endocrine and Metabolic Diseases, China National Research Center for Metabolic Diseases, Ruijin Hospital, Shanghai JiaoTong University School of Medicine (SJTUSM), Shanghai, China

Correspondence should be addressed to G Ning or Q Ma: gning@sibs.ac.cn or qinyunma@126.com

\begin{abstract}
Appetite is tightly controlled by neural and hormonal signals in animals. In general, steroid receptor coactivator 1 (SRC1) enhances steroid hormone signalling in energy balance and serves as a common coactivator of several steroid receptors, such as oestrogen and glucocorticoid receptors. However, the key roles of SRC1 in energy balance remain largely unknown. We first confirmed that SRC1 is abundantly expressed in the hypothalamic arcuate nucleus (ARC), which is a critical centre for regulating feeding and energy balance; it is further co-localised with agouti-related protein and proopiomelanocortin neurons in the arcuate nucleus. Interestingly, local SRC1 expression changes with the transition between sufficiency and deficiency of food supply. To identify its direct role in appetite regulation, we repressed SRC1 expression in the hypothalamic ARC using lentivirus shRNA and found that SRC1 deficiency significantly promoted food intake and body weight gain, particularly in mice fed with a high-fat diet. We also found the activation of the AMP-activated protein kinase (AMPK) signalling pathway due to SRC1 deficiency. Thus, our results suggest that SRC1 in the ARC regulates appetite and body weight and that AMPK signalling is involved in this process. We believe that our study results have important implications for recognising the overlapping and integrating effects of several steroid hormones/receptors on accurate appetite regulation in future studies.
\end{abstract}

Key Words
- SRC1
knockdown
- food intake
- hypothalamic arcuate
nucleus
- body weight

\section{Introduction}

Understanding how an organism regulates food intake is fundamental for treating pathological conditions, such as obesity, anorexia nervosa and bulimia nervosa, in which the normal appetite regulation is disturbed (Fetissov 2016). The increasing incidence of obesity reflects the lack of efficient treatments and highlight our insufficient knowledge about the mechanisms underlying feeding behaviour (Aigner et al. 2011, Gautron et al. 2015).
The hypothalamus, particularly the arcuate nucleus (ARC), plays a crucial role in regulating feeding and energy balance. Increased electrical activity in orexigenic agouti-related protein (AgRP) neurons rapidly induces voracious feeding behaviour and silences anorexigenic proopiomelanocortin (POMC) neurons, which inhibit feeding (Atasoy et al. 2012). These two types of neurons are located in the ARC. They behave as interoceptive 
sensory neurons and modulate their electric activity in response to hormonal signals of the metabolic state, such as insulin, leptin and ghrelin levels. Moreover, together with steroid receptors, such as oestrogen receptor alpha $(\mathrm{ER} \alpha)$ and glucocorticoid receptor (GR), they form a core circuit that regulates food intake and energy expenditure.

Steroid receptor coactivator 1 (SRC1, also known as NCOA1) is a member of the p160 steroid receptor coactivator (SRC) family, which also includes SRC2 (NCOA2, TIF2 or GRIP1) and SRC3 (AIB1, p/CIP, ACTR, RAC3 or NCOA3) (York \& O'Malley 2010, Stashi et al. 2014). SRC1 can interact with and co-activate the nuclear receptors (NRs) in the presence of hormones; these NRs include progesterone receptor, GR, ER $\alpha$, thyroid receptor, retinoid $\mathrm{X}$ receptor and peroxisome proliferator-activated receptor $\gamma$ (PPAR $\gamma$ ) (Onate et al. 1995, Zhu et al. 1996). SRC1 can also co-activate other non-steroidal transcription factors, such as AP1, serum response factor and NFKB (Lee et al. 1998, Na et al. 1998). SRC1 is currently the most studied SRC family member in the central nervous system (CNS). It is widely expressed throughout the brain and is present at high levels in the cortex, hippocampus and olfactory bulbs (Meijer et al. 2000). In the brain, it regulates moderate motor functions and the anxiety response (Nishihara et al. 2003) as well as sexual and behavioural development (Auger et al. 2000, Apostolakis et al. 2002) and the hypothalamic-pituitary-adrenal axis (Winnay et al. 2006, Lachize et al. 2009). SRC1 has been identified as a key pleiotropic regulator of energy balance. SRC1knockout mice exhibit decreased energy expenditure with increased adiposity (Picard et al. 2002). SRC1 is also a key coordinator of the hepatic gluconeogenic programme in vivo; for example, in one study, SRC1-knockout mice displayed hypoglycaemia secondary to a deficit in hepatic glucose production (Louet et al. 2010). In particular, SRC1 is functionally required to mediate the anti-obesity effects of hypothalamic oestrogen-ER $\alpha$ signals (Zhu et al. 2013).

The direct metabolic regulator function of SRC1 in the hypothalamic ARC is poorly understood. Our study found that SRC1 expression in the ARC fluctuated in response to the external food supply. Importantly, we further demonstrated that the targeted knockdown of SRC1 in the ARC regulated food intake and body weight without affecting energy expenditure or adaptive thermogenesis. In addition, the hypothalamus AMP-activated protein kinase (AMPK) signalling pathway was activated in the ARC SRC1-knockdown mice. Our study illustrated the direct role of hypothalamic SRC1 on energy balance, which may provide novel insights for understanding the development of obesity and other metabolic disorders.

\section{Materials and methods}

\section{Animals}

This study used male C57BL/6J mice obtained from Shanghai Laboratory Animal Co. Ltd. (Shanghai, China) and AgRP-Cre, POMC-Cre and rosa26-EGFP mice obtained from Jackson Laboratory. All animals were individually housed in a temperature-controlled colony room $\left(22-24^{\circ} \mathrm{C}\right)$ under a 12:12-h light-dark cycle with ad libitum access to a standard laboratory rodent chow diet $(1.404 \mathrm{kcal} / \mathrm{g}$, SLAC Laboratory Animal Co. Ltd., Shanghai, China) or a highfat diet (HFD) with $60 \% \mathrm{kcal} \%$ fat $(5.24 \mathrm{kcal} / \mathrm{g}$, Research Diets Inc.). The AgRP-Cre and POMC-Cre mice received an injection of recombinant adeno-associated virus in the ARC (AAV-GFP, purchased from OBiO Technology, Shanghai, China), which contained an inverted GFP reporter with a loxP site at both ends; the two loxP sites displayed opposite orientations so that the GFP reporter could only be expressed in the presence of Cre recombinase. Consequently, GFP could be specifically expressed in AgRP neurons (AgRP-GFP mice) or POMC neurons (POMC-GFP mice). These experiments were conducted in accordance with the guidelines of the Institutional Animal Care and Use Committee of Shanghai Jiaotong University and were approved by the Institutional Animal Care and Use Committee of Shanghai Jiaotong University.

\section{Lentivirus-mediated knockdown of SRC1 expression in the GT1-7 cell line and the ARC of mice}

GT1-7 cells grown in 6-well or 24-well plates were maintained in Dulbecco's modified Eagle's medium, containing $10 \%$ foetal bovine serum, $2 \mathrm{mM}$ L-glutamine and $1 \times$ penicillin/streptomycin, in a humidified incubator with $5 \% \mathrm{CO}_{2}$ at $37^{\circ} \mathrm{C}$. All reagents were obtained from GIBCO. The GT1-7 cell line was kindly provided by Michael Weiner at the University of California-San Francisco (San Francisco, CA, USA).

The lentivirus was purchased from OBiO Technology (Shanghai, China). The virus titre was $1 \times 10^{11} \mathrm{pfu}$. To examine the effects of the lentivirus on SRC1 gene expression, we firstly infected GT1-7 cells with shRNAGFP and shRNA-SRC; multiplicity of infection (MOI) of the virus was 100. The chosen shRNA sequence for Src1 was 5'-CAGCAGCTACTGACTGAATAACT CGAGTTATTCAGTCAGTAGCTGCTGTTTTT-3', and the sequence for NT (the negative control) was 5'-AAACTACCGTTGTTATAGGTGCTCGAGCACCTATA ACAACGGTAGTTT-3'. After being infected with the virus for $48 \mathrm{~h}$, the cells were collected for further real-time 
PCR or western blot analysis. Meanwhile, adult C57BL/6 mice were injected with lentivirus to deliver shRNA-SRC into one side of the ARC and shRNA-GFP into the other side in the same mice. One week later, the mice were transcardially perfused with 4\% PFA under anaesthesia, and their brains were removed to assess for intra-ARC deliveries by immunostaining.

\section{Male C57BL/6J mice injected with the virus in the hypothalamic ARC}

After determining the effects of shRNA-SRC on SRC1 expression, male C57BL/6J mice fed an HFD or a normal chow diet (NCD) was randomly assigned to receive bilateral ARC injections of shRNA-GFP or shRNA-SRC. In the HFD group, the mice received an HFD for 1 month prior to injection. The bilateral injections to the ARC were directed using an ultra-precise stereotaxy (Kopf Instruments, Tujunga, CA, USA) with coordinates of $1.5 \mathrm{~mm}$ posterior to the bregma, $5.8 \mathrm{~mm}$ below the surface of the skull and $0.4 \mathrm{~mm}$ lateral to the midline. Purified lentivirus in artificial cerebrospinal fluid was injected at a rate of $0.1 \mu \mathrm{L} / \mathrm{min}$ over $10 \mathrm{~min}$ through a 33-gauge injector connected to a Hamilton syringe and an infusion pump. The injector remained in place for an additional $5 \mathrm{~min}$ before removal. The same method was used to create AgRP-GFP and POMC-GFP mice.

\section{Effects of the ARC SRC1 knockdown on food intake and body weight}

Following the ARC injection of shRNA-SRC or shRNAGFP, food intake and body weight of the individually housed mice were assessed daily. The food intake data were summed to obtain the accumulated food intake. Ten to twelve days after the viral injection, the mice were killed, and their blood and tissues (the entire hypothalamus, liver and adipose tissue) were collected. The adipose tissues were weighed.

\section{Quantitative real-time PCR}

Total RNA was extracted from the cells or tissues using TRIzol reagent (Invitrogen) following the manufacturer's instructions. Briefly, $1 \mu \mathrm{g}$ RNA was transcribed to complementary DNA using the Reverse Transcription System (Promega). Real-time PCR was performed with the LC480 system (Roche) using SYBER Green Supermix (Takara). The data were normalised to 36b4, and the results were expressed as fold changes of the threshold cycle $(\mathrm{Ct})$ values relative to the controls. All the primer sets were designed to span at least one intron to avoid genomic DNA-derived amplification. The following primers of Src1 were used: forward primer, 5'-GCCAGCGCTATGAAGTAATGCA-3' and reverse primer, 5'-CGAGGTAATCGTCGAGCAATACAA-3'.

\section{Western blot analysis}

Total protein lysates from the cells and tissues were prepared using RIPA reagent (Biocolors, Shanghai, China). Briefly, $20 \mu \mathrm{g}$ protein samples were separated using $4-12 \%$ SDS-PAGE and transferred onto a PVDF membrane. The membrane was then incubated with primary antibodies (anti-p-AKT, anti-total-AKT, anti-p-mTOR, anti-total-mTOR, anti-p-S6K1, anti-total-S6K, anti-p-S6 and anti-total-S6, all of which were obtained from Cell Signaling Technology), followed by incubation with horseradish peroxidaselabelled donkey anti-goat antibodies (Cell Signaling Technology) and detection using an Immobilon Western Chemiluminescent HRP Substrate Kit (Millipore).

\section{Heart perfusion and immunostaining}

The mice were transcardially perfused with 4\% PFA under anaesthesia, and their brains were removed, post-fixed in 4\% PFA and infiltrated with $20-30 \%$ sucrose. Brain sections ( $15 \mu \mathrm{m}$ thickness) across the hypothalamus were blocked with the serum of appropriate species, penetrated with $0.2 \%$ Triton $\mathrm{X}-100$, treated with primary antibodies (anti-SRC1: Santa Cruz Biotechnology; anti-GFP and anti-ER $\alpha$ : Cell Signalling Technology) and then subjected to reaction with fluorescence-conjugated secondary antibodies (Invitrogen). A coverslip was then placed over the samples prior to examination under a laser scan confocal microscope (Zeiss) and a fluorescent microscope (Olympus).

\section{Statistical analysis}

All results are presented as mean \pm standard error of the mean (s.e.m.). $P$ values were calculated by unpaired Student's $t$-test, one-way ANOVA and two-way ANOVA.

\section{Results}

\section{SRC1 is expressed in the hypothalamic ARC and} oscillates with the nutritional condition

Consistent with the results of a previous study (Zhu et al. 2013), we found that SRC1 was abundantly expressed in 
A
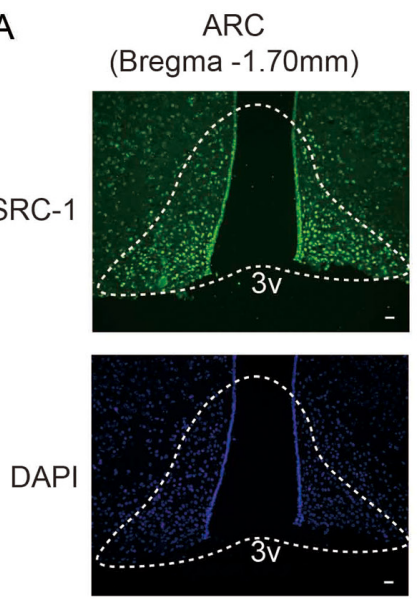

PVN (Bregma -0.70mm)
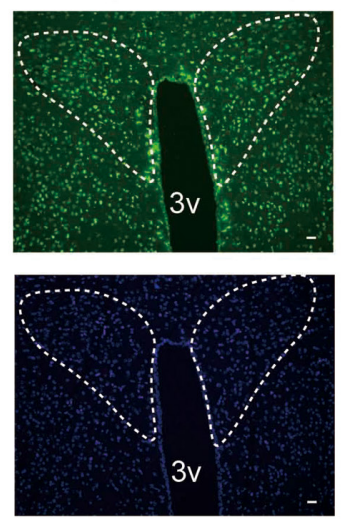

$\mathrm{VMH}$

(Bregma -1.46mm)
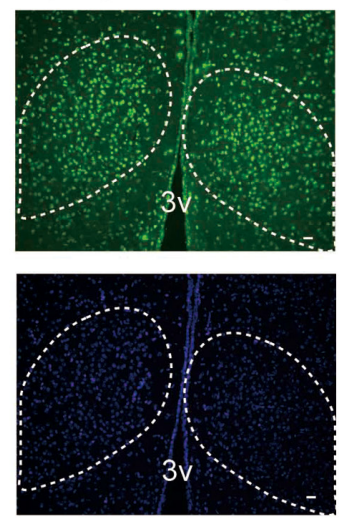

\section{Figure 1}

Distribution of SRC1 in mouse hypothalamic nucleus. (A) A representative immunofluorescent staining for SRC1 (green) in mice. 10 sections from three mice, all images were generated by fluorescent microscope. 3V, third ventricle. Scale bar, $100 \mu \mathrm{m}$. A full colour version of this figure is available at https://doi.org/10.1530/JME-18-0075. the mouse hypothalamic nuclei, as shown in Fig. 1, mainly the paraventricular nucleus (PVN), ventromedial nucleus (VMH) and ARC. Because POMC and NPY/AgRP neurons in the ARC play a central role in feeding and energy homeostasis, we further determined the SRC1 expression in POMC and NPY/AgRP neurons and found that SRC1 was expressed in both types of neurons (Supplementary Fig. $1 \mathrm{~A}$ and $\mathrm{B}$, see section on supplementary data given at the end of this article).

The hypothalamic regulators that affect energy homeostasis are generally regulated by various nutritionsupply statuses, such as chow or HFD and fasting or feeding (Morton et al. 2006). To evaluate the potential biological functions of hypothalamic SRC1 in energy balance, we first assessed SRC1 expression under various nutritional conditions (Fig. 2). We found that the mRNA expression of hypothalamic Src1 was significantly reduced by approximately $60 \%$ after the mice were fasted for $24 \mathrm{~h}$; this expression level was partly restored by refeeding (Fig. 2A). Furthermore, hypothalamic Src1 expression decreased by up to $40 \%$ in mice on a long-term (20 weeks) HFD (Fig. 2B). These results suggested that hypothalamic Src1 senses the body energy balance.

\section{ARC-specific SRC1 knockdown promotes food intake and increases body weight}

To clarify the direct roles of hypothalamic SRC1 on feeding, we next constructed Src1 shRNA lentivirus (shRNA-SRC) to knockdown SRC1 in the ARC. We also constructed a lentivirus with GFP to serve as the control (shRNA-GFP); the knockdown efficiency of this virus was confirmed in hypothalamic GT1-7 cells and tissues (Fig. 3A, B and C). The knockdown efficiency in the HFDfed mice was slightly higher than that in the NCD-fed mice (Fig. 3C), but no significance was observed. To ensure that the lentivirus did not cause changes in the expression of other members of the SRC family, we examined the expression of $\mathrm{Src} 2$ and $\mathrm{Src} 3$ in the hypothalamus and found no significant differences (Fig. 3D). Compared with the shRNA-GFP injection side in the hypothalamic ARC, a significant reduction in the SRC1 positive cells was observed in the shRNA-SRC injection side in both NCD (Fig. 3E and F) and HFD (Fig. 3H and I). In addition, counting of the number of positively stained SRC1 cells in the ARC of both the shRNA-GFP and shRNA-SRC groups also revealed a significant reduction in SRC1 in the shRNA-SRC group (Fig. 3G and J). The ARC is commonly considered as the 'headquarters' for feeding activity; thus, we first examined the daily food intake of mice with an SRC1 deficiency. The daily food intake of the shRNA-SRC mice on NCD increased by approximately $12 \%$ from day 8 to 10 compared with that of the shRNA-GFP control mice with NCD (Fig. 4A). Meanwhile, the accumulative food
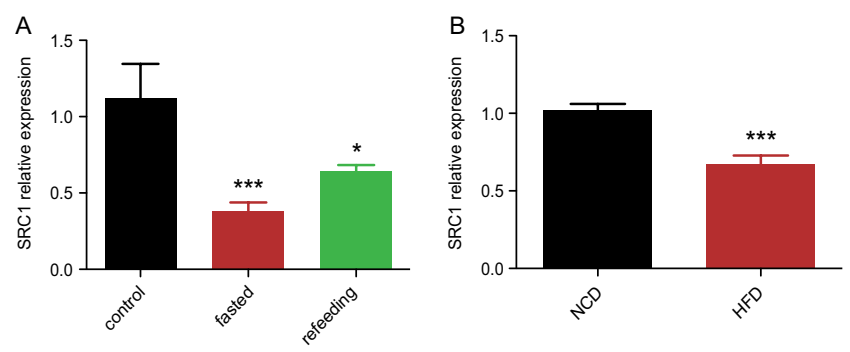

Figure 2

Expression of hypothalamic SRC1 in different nutritional conditions. (A) Hypothalamic Src1 expression was reduced by $24 \mathrm{~h}$ of fasting and partly restored by $6 \mathrm{~h}$ of refeeding. (B) The hypothalamic Src1 expression in mice fed with an HFD for 6 months was reduced compared with that in mice fed with NCD. mRNA was measured by quantitative real-time PCR and relative to $36 b 4$. Data are presented as mean \pm S.E.M. and were analysed using one-way ANOVA (A) and Student's $t$-test (B). ${ }^{*} P<0.05, * * * P<0.001$, $n=10(\mathrm{~A})$ or $8(\mathrm{~B})$ mice per group. A full colour version of this figure is available at https://doi.org/10.1530/JME-18-0075. 
A
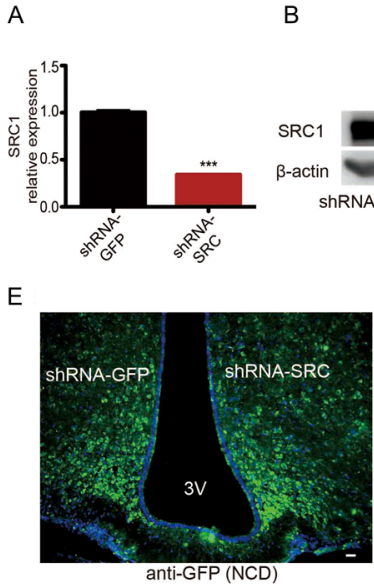

$\mathrm{H}$

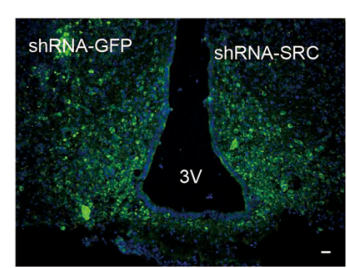

anti-GFP (HFD)

B

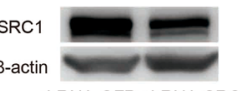

ShRNA-GFP ShRNA-SRC

$\mathrm{F}$

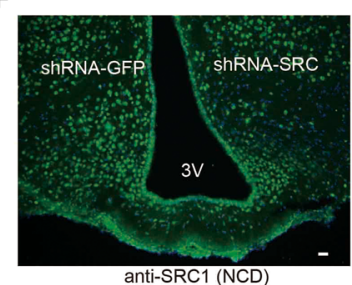

I

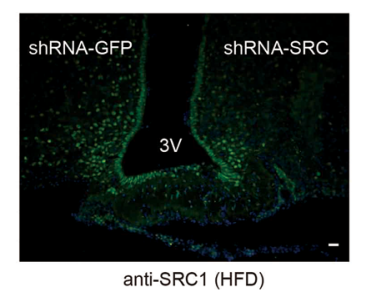

C

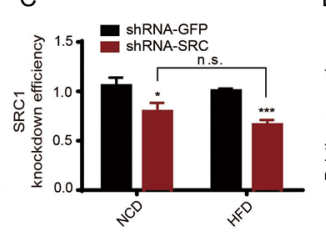

G

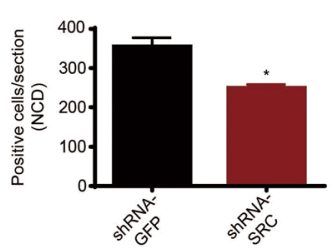

J

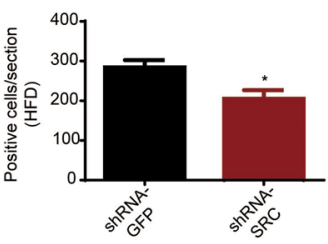

Figure 3

Construction of the $\operatorname{Src} 1$ shRNA lentivirus and efficiency of SRC1 in the ARC and GT1-7 cell line. (A and B) Lentivirus-mediated Src1 knockdown in the GT1-7 cell line. Src1 mRNA was measured by quantitative real-time PCR and relative to $36 b 4$ (A). The SRC1 protein level was tested by western blotting, and $\beta$-actin levels were shown as the loading control (B). $\mathrm{MOI}=60 \mathrm{pfu}$ number/cell, $n=4$ per group. (C and D) The hypothalamic $\operatorname{Src} 1$ (C), Src2 and SrC3 (D) levels after bilateral ARC injection of the $\operatorname{Src} 1$ lentivirus (shRNA-SRC) compared with those after injection of shRNA-GFP examined by real-time PCR ( $n=11-16$ per group). (E, F, H and I) SRC1 and GFP staining in the ARC of mice receiving unilateral $A R C$ injections in both $\mathrm{NCD}$ ( $\mathrm{E}$ and $\mathrm{F}$ ) and HFD ( $\mathrm{H}$ and $\mathrm{I})$. The mice were injected with shRNA-GFP into one side of the ARC and shRNA-SRC into the other side. Brain sections across the ARC were stained for GFP ( $E$ and $H$ ) or SRC1 ( $F$ and I) antibodies and observed by fluorescence microscopy. ( $G$ and J) Immunostaining quantification of SRC1-positive cells in the ARC ( $n=3$ per group, 8-10 sections per sample). $3 V$, third ventricle. Scale bar, $100 \mu \mathrm{m}$. Data are presented as mean \pm s.E.M. and were analysed using Student's $t$-test and two-way ANOVA (C). $* P<0.05, * * * P<0.001$. A full colour version of this figure is available at https://doi. org/10.1530/JME-18-0075. intake also showed an increasing trend ( $P=0.07$, Fig. 4B). Notably, both the daily food intake and the accumulative food intake significantly increased in the SRC1-knockdown mice with HFD (Fig. 4C and D). More food was consumed by the shRNA-SRC mice after day 8 . These data suggested that a SRC1 deficiency in the hypothalamic ARC could robustly increase food intake under both NCD and HFD.

The imbalance between caloric intake and energy expenditure results in body weight gain and a series of metabolic disorders. Because SRC1 knockdown in the hypothalamic ARC caused hyperphagia, we further detected the effects of SRC1 knockdown on body weight. We found that the body weight of shRNA-SRC mice increased more significantly than that of shRNAGFP mice, under both NCD (Fig. 5A) and HFD (Fig. 5B). Moreover, SRC1-deficient mice fed with HFD exhibited a more marked body weight gain; the fat pad weights of the shRNA-SRC mice increased but were not significantly different from those of the shRNA-GFP mice (Fig. 5C).

The body weight increase may be due to the increased food intake and/or decreased energy expenditure. Furthermore, a previous study has suggested that global SRC1-knockout mice have impaired adaptive thermogenesis and diminished energy expenditure (Picard et al. 2002). To investigate the effects of SRC1 deficiency on energy expenditure, we further measured oxygen consumption $\left(\mathrm{VO}_{2}\right)$ and carbon dioxide production $\left(\mathrm{VCO}_{2}\right)$ by indirect calorimetry 8 days after the shRNASRC and shRNA-GFP treatments and found no significant differences between these two groups (Fig. 5D and E). In addition, the mRNA expression of key thermogenic

A
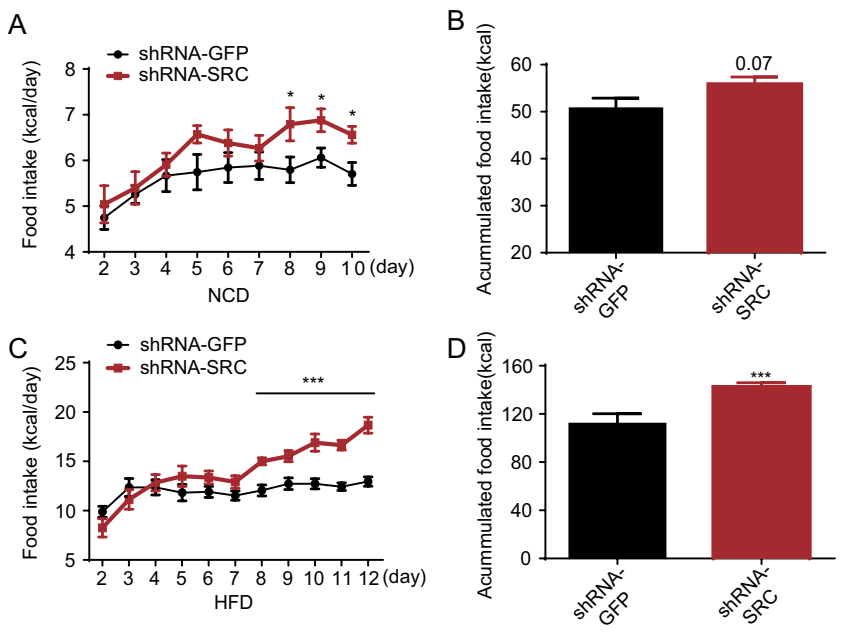

Figure 4

The ARC SRC1 knockdown regulates food intake. (A, B, C and D) Daily food consumption and accumulative food intake between the shRNA-SRC and shRNA-GFP mice that were fed with NCD (A and B) and HFD (C and D). Data are presented as mean \pm S.E.M. and were analysed using Student's $t$-test. ${ }^{*} P<0.05,{ }^{*} P<0.01, n=7-11$ per group. A full colour version of this figure is available at https://doi.org/10.1530/JME-18-0075. 

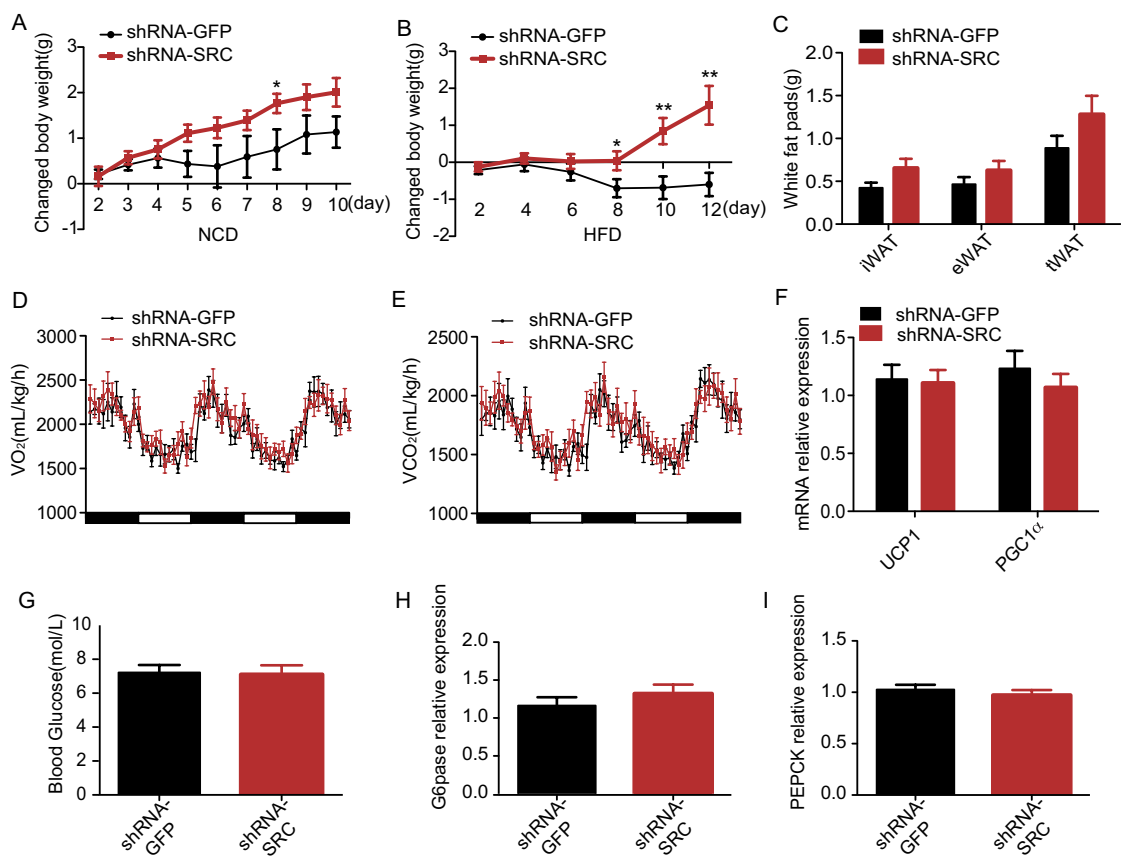

\section{Figure 5}

The ARC SRC1 knockdown regulates body weight but not via the mechanisms of energy expenditure or glucose production. (A and B) Changes in the body weight of shRNA-SRC and shRNA-GFP mice that were fed with NCD (A) and HFD (B). The change in body weight was based on the body weights on the first day after injection. (C) The weight of the white fat pads from shRNA-SRC and shRNA-GFP mice that were fed with HFD. (D and E) Oxygen consumption $\left(\mathrm{VO}_{2}\right)(\mathrm{D})$ and carbon dioxide production $\left(\mathrm{VCO}_{2}\right)(\mathrm{E})$ analysed by indirect calorimetry in shRNA-SRC and shRNA-GFP mice that were fed with HFD presented over three consecutive 12-h dark and two 12-h light cycles. (F) The Ucp1 and Pgc1 $\alpha$ expression in the brown adipose tissue of shRNA-SRC and shRNA-GFP mice fed with HFD was measured by quantitative PCR and relative to 36b4. (G) Blood glucose levels of shRNA-SRC and shRNA-GFP mice fed with HFD after fasting overnight. ( $\mathrm{H}$ and I) The G6pase $(\mathrm{H})$ and Pepck (I) mRNA expression in the liver of shRNA-SRC and shRNA-GFP mice measured by quantitative real-time PCR. eWAT, epididymal adipose tissue; iWAT, inguinal adipose tissue; tWAT, total adipose tissue. Data are presented as mean \pm S.E.M. and were analysed using Student's $t$-test. ${ }^{*} P<0.05$, $\star * P<0.01, n=7-11$ per group. A full colour version of this figure is available at https://doi. org/10.1530/JME-18-0075. genes, such as Ucp1 and $P g c 1 \alpha$, showed no significant change in brown adipose tissues (Fig. 5F). Moreover, we found that no difference in fasting blood glucose between these two groups (Fig. 5G) and no change in the expression of genes associated with gluconeogenesis (Fig. 5H and I). These data suggest that the SRC1 deficiency in the hypothalamic ARC could increase body weight by promoting food intake without affecting energy expenditure or glucose production.

\section{Hormones and signalling pathway changes in the ARC SRC1-knockdown mice}

Leptin and insulin are two key hormones involved in regulating feeding and energy balance. In our study, both serum leptin and insulin levels increased almost significantly in the shRNA-SRC mice compared with those in the shRNA-GFP mice $(P=0.05$ and 0.06 for leptin and insulin, respectively Fig. $6 \mathrm{~A}$ and $\mathrm{B})$. These results indicate that the leptin and insulin action was reduced and could be secondary to body weight gain due to SRC1 deficiency in the ARC.

A previous study has shown that the interaction between ER $\alpha$ and SRC1 can prevent body weight gain in female mice (Zhu et al. 2013). We found that the ER $\alpha$ expression in the ARC was reduced (Fig. 6C and D). AMPK and mTOR signalling, as the two fuel-sensing pathways, also exhibit an important role in regulating feeding behaviour (Minokoshi et al. 2004, Cota et al. 2006). Thus, we further examined the activation of these two pathways and found that the AMPK signalling pathway was activated when SRC1 was knocked down in the hypothalamic ARC (Fig. 6E and F). However, phosphorylated P70s6 kinase (S6K) and S6 of the mTOR signalling pathway showed no significant differences in the SRC1-deficient mice (Fig. 6G and $\mathrm{H}$ ). Our data suggest that SRC1 deficiency in the ARC increased food intake and body weight by downregulating the ER $\alpha$ expression and activating the AMPK signalling pathway (Fig. 7).

\section{Discussion}

Because of the worldwide epidemic burden of obesity and overweight (Afshin et al. 2017), it is important to further define the regulatory principles underlying the control of body weight and to provide novel targets for therapeutic intervention. Over the past decades, genetic studies on obesity have emphasised the roles of the CNS and, more specifically, the hypothalamus in the control of weight homeostasis by governing food intake and 
A

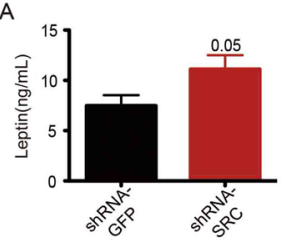

C

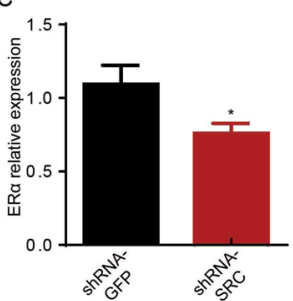

E

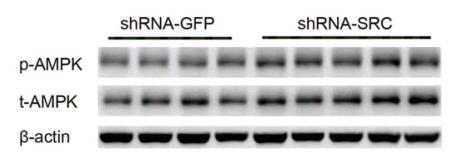

G

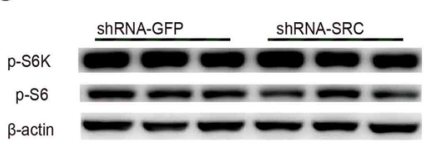

B
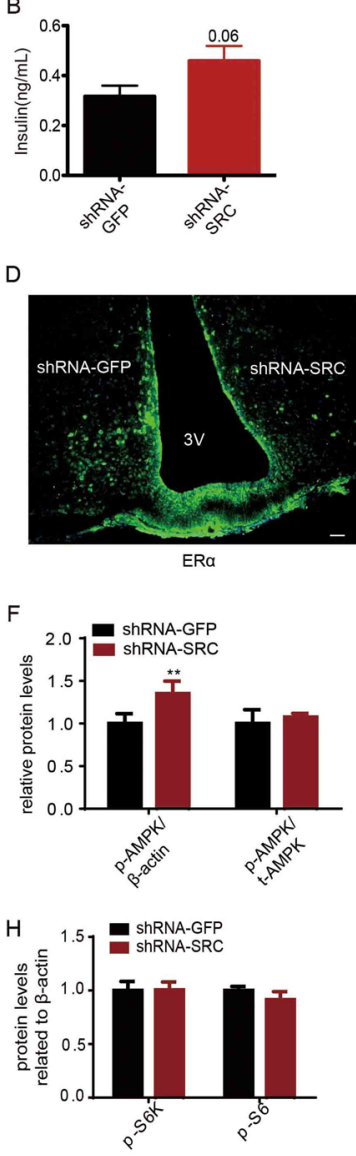

\section{Figure 6}

SRC1 knockdown in the ARC reduces ER $\alpha$ but activates the hypothalamic AMPK signalling pathway. (A and B) Serum leptin levels (A) and insulin levels (B) of shRNA-SRC and shRNA-GFP mice fed with HFD. (C) The mRNA expression of $E r \alpha$ in the hypothalamus. (D) ER $\alpha$ staining in the ARC of mice that received unilateral ARC injections. The mice were injected with shRNA-GFP into one side of the ARC and shRNA-SRC into the other side. The images were generated by fluorescence microscopy; nine sections from three mice were imaged, and one representative image was chosen. $3 \mathrm{~V}$, third ventricle. Scale bar, $100 \mu \mathrm{m}$. (E and F) AMPK phosphorylation and protein expression of the hypothalamus of shRNA-SRC or shRNA-GFP mice fed with HFD. Protein levels were measured by western blotting, and $\beta$-actin levels are shown as the loading control. The phosphorylated AMPK level of the hypothalamus (E) was quantified using Image J relative to $\beta$-actin and t-AMPK (F). (G and H) Hypothalamic phosphorylated S6K, S6, and $\beta$-actin protein levels of shRNA-SRC mice and shRNA-GFP mice with HFD were measured by western blotting, $\beta$-actin levels are shown as the loading control (G), The phosphorylated S6K and S6 level was quantified using Image J relative to $\beta$-actin $(H)$. Data are presented as mean \pm S.E.M. and were analysed using Student's $t$-test. ${ }^{*} P<0.05,{ }^{*} * P<0.01, n=13$ per group ( $A, B$ and $D), 4-5$ per group $(F$ and $H)$. A full colour version of this figure is available at https://doi.org/10.1530/JME-18-0075.

energy expenditure (Morton et al. 2006). Further, the hypothalamic ARC is central to coordinating food intake with the energy state of the organism (Denis et al. 2014). Further exploration of the novel potential regulators that control feeding behaviour, sense the nutrient state in the ARC and regulate body weight is warranted.

The transcription coactivator SRC1 is involved in energy expenditure in adipose tissue and the liver (Picard et al. 2002, Louet et al. 2010), whereas SRC1 in the CNS is mainly involved in neuron plasticity, neural stem cell differentiation and motor learning (Nishihara et al. 2003, Zhang et al. 2011). Although one study found that SRC1 mediated the anti-obesity effects of hypothalamic oestrogen-ER $\alpha$ signals in females (Zhu et al. 2013), the direct roles of nucleus-specific SRC1 on energy balance remain unclear. Our study provided evidence that SRC1 in the ARC regulates the appetite to affect body weight. We found that SRC1 was abundantly expressed in the hypothalamus, including the ARC. More interestingly, hypothalamic SRC1 expression showed an evident oscillation as per nutritional fluctuations. SRC1 was reduced in both fasting and HFD conditions. In the fasting condition, insulin and leptin levels are low. However, in an HFD condition, the body has insulin resistance and leptin resistance (Burchfield et al. 2018). Therefore, the hypothalamus cannot obtain sufficient insulin and leptin signals, which the hypothalamus then associates the insufficient levels of these hormones with hunger. We speculated that SRC1 in the ARC might play an integrated sensor role in nutrition status, according to our results, but we need more data to prove it in future. Importantly, the targeted knockdown of SRC1 in the ARC increased food consumption and further promoted body weight gain, particularly under the HFD stimulation. Although the global SRC1-knockout mice have previously shown a decrease in energy expenditure (Picard et al. 2002), no difference was observed in energy consumption between the ARC SRC1-deficient mice and the control mice in our study. Thus, the ARC-specific SRC1 deficiency increased energy intake but did not reduce energy expenditure. Taken together, these results suggest that peripheral and central SRC1 act together to increase energy storage and promote body weight gain.

SRC1 interacts with a few NRs, particularly hormone receptors, such as ER, AR and GR and fatty acid receptors, such as PPARs, which have been reported to control feeding and energy balance in the hypothalamus (Kocalis et al. 2012, Zhu et al. 2013, Sefton et al. 2016, Morford et al. 2017). VMH microinjections of oestradiol benzoate (E2) decrease food consumption in rats (Wade \& Zucker 1970, Frank et al. 2014). Glucocorticoids can regulate anatomical and electrophysiological properties of POMC and NPY/AgRP neurons in the ARC (Gyengesi et al. 2010). Blocking the endogenous activation of the CNS PPAR $\gamma$ with pharmacological antagonists or genetic deletion leads to a negative energy balance (Ryan et al. 2011). 


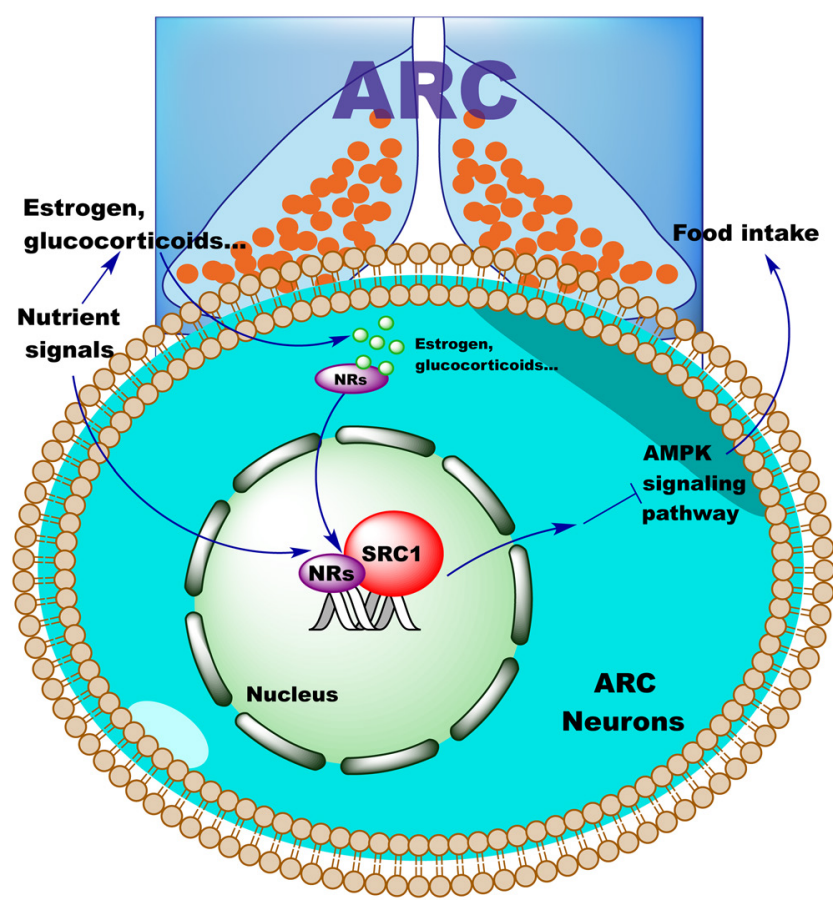

Figure 7

Schematic representation of the role of SRC1 in the ARC. SRC1 in the ARC could be regulated by both hormone signals and nutrient signals. After interacting with some NRs, SRC1 suppressed AMPK phosphorylation and protein expression, thus modulating food intake. A full colour version of this figure is available at https://doi.org/10.1530/JME-18-0075.

It was hypothesised that SRC1 in the ARC acts as an integrated sensor to produce a final readout on appetite by co-activating with NRs. It will be interesting to determine whether more NR factors are involved with the complex. The modulation of feeding and energy balance of other NRs interacting with SRC1 in the ARC requires further investigation.

Leptin and insulin receptors, the dominant hormone receptors in energy balance, are expressed in hypothalamic neurons. Several studies have highlighted their importance in mediating anorexigenic and glucoregulatory effects, specifically on ARC neurons. In this study, the knockdown of hypothalamic SRC1 increased food intake but did not change glucose homeostasis associated with higher plasma leptin and insulin levels. It was postulated that the trends in leptin and insulin levels were secondary to the increased food intake and that their anorexigenic effects were reduced when hypothalamic SRC1 was deficient.

A previous study has shown that the central administration of ER $\alpha$ agonist reduces food intake and body weight gain by promoting the physical interaction between SRC1 and ER $\alpha$ in the hypothalamus (Zhu et al. 2013). Some evidence has indicated that ERo signalling interacts with the AMPK and mTOR pathways (Dhillon \& Belsham 2011, Shrivastav et al. 2014, Daurio et al. 2016, Guo et al. 2017), which are the well-defined nutrition sensors in the peripheral and central tissues (Minokoshi et al. 2004, Cota et al. 2006, Fernández-Veledo et al. 2013). Thus, these two pathways were carefully evaluated after the knockdown of hypothalamic SRC1. We found that SRC1 deficiency activated the AMPK pathway but not the mTOR signalling pathway in vivo. Peripheral AMPK monitors the cellular energy status as a 'fuel gauge' of the AMP/ATP ratio, and hypothalamic AMPK regulates food intake by responding to hormonal and nutrient signals (Hardie et al. 2003, Minokoshi et al. 2004). These findings further support that SRC1 in the ARC may function as a sensor in the second line of hormonal and nutrient signals. It would be informative to investigate the longterm effects of SRC1 in the hypothalamus with various neuron-specific knockout mouse models.

It is regrettable that the SRC1 overexpression lentivirus construction failed because the molecular weight of SRC1 is too high. Regardless of this limitation, our study indicates that SRC1 in the ARC plays a significant role in appetite regulation and further obesity development and that it might function as an integrating sensor of various nutrition signals, including steroid hormone levels, nonsteroid hormone levels and neural energy status. These results provide some basis for exploring the appetite network node and obesity drug targets in future studies.

\section{Supplementary data}

This is linked to the online version of the paper at https://doi.org/10.1530/ JME-18-0075.

\section{Declaration of interest}

The authors declare that there is no conflict of interest that could be perceived as prejudicing the impartiality of the research reported.

\section{Funding}

This work was supported by grants from the National Natural Science Foundation of China (No. 81270867 and No. 81471061).

\section{Author contribution statement}

G N and Q M designed the studies. Q L and Q M performed all experimental procedures. J W, R L, S Z, Y Y and Y H performed the experimental data analyses. B G, S J and P L performed the tissue harvests. Q L and Q M wrote the manuscript. All authors contributed to the discussion, revised the manuscript and approved the final version. $\mathrm{G} \mathrm{N}$ is responsible for the integrity of the work as a whole. 


\section{References}

Afshin A, Forouzanfar M, Reitsma M, Sur P, Estep K, Lee A, Marczak L, Mokdad A, Moradi-Lakeh M, Naghavi M, et al. 2017 Health effects of overweight and obesity in 195 countries over 25 years. New England Journal of Medicine 377 13-27. (https://doi.org/10.1056/ NEJMoa1614362)

Aigner M, Treasure J, Kaye W \& Kasper S 2011 World Federation of Societies of Biological Psychiatry (WFSBP) guidelines for the pharmacological treatment of eating disorders. World Journal of Biological Psychiatry 12 400-443. (https://doi.org/10.3109/15622975.2 011.602720)

Apostolakis EM, Ramamurphy M, Zhou D, Onate S \& O'Malley BW 2002 Acute disruption of select steroid receptor coactivators prevents reproductive behavior in rats and unmasks genetic adaptation in knockout mice. Molecular Endocrinology 16 1511-1523. (https://doi. org/10.1210/mend.16.7.0877)

Atasoy D, Betley JN, Su HH \& Sternson SM 2012 Deconstruction of a neural circuit for hunger. Nature 488 172-177. (https://doi. org/10.1038/nature11270)

Auger AP, Tetel MJ \& McCarthy MM 2000 Steroid receptor coactivator-1 (SRC-1) mediates the development of sex-specific brain morphology and behavior. PNAS 97 7551-7555. (https://doi.org/10.1073/ pnas.97.13.7551)

Burchfield JG, Kebede MA, Meoli CC, Stockli J, Whitworth PT, Wright AL, Hoffman NJ, Minard AY, Ma X, Krycer JR, et al. 2018 High dietary fat and sucrose results in an extensive and timedependent deterioration in health of multiple physiological systems in mice. Journal of Biological Chemistry 293 5731-5745. (https://doi. org/10.1074/jbc.RA117.000808)

Cota D, Proulx K, Smith K, Kozma S, Thomas G, Woods S \& Seeley R 2006 Hypothalamic mTOR signaling regulates food intake. Science 312 927-930. (https://doi.org/10.1126/science.1124147)

Daurio N, Tuttle S, Worth A, Song E, Davis J, Snyder N, Blair I \& Koumenis C 2016 AMPK activation and metabolic reprogramming by tamoxifen through estrogen receptor-independent mechanisms suggests new uses for this therapeutic modality in cancer treatment. Cancer Research 76 3295-3306. (https://doi.org/10.1158/0008-5472. CAN-15-2197)

Denis R, Joly-Amado A, Cansell C, Castel J, Martinez S, Delbes A \& Luquet S 2014 Central orchestration of peripheral nutrient partitioning and substrate utilization: implications for the metabolic syndrome. Diabetes and Metabolism 40 191-197. (https://doi. org/10.1016/j.diabet.2013.11.002)

Dhillon S \& Belsham D 2011 Estrogen inhibits NPY secretion through membrane-associated estrogen receptor (ER)- $\alpha$ in clonal, immortalized hypothalamic neurons. International Journal of Obesity 35 198-207. (https://doi.org/10.1038/ijo.2010.124)

Fernández-Veledo S, Vázquez-Carballo A, Vila-Bedmar R, CeperueloMallafré V \& Vendrell J 2013 Role of energy- and nutrient-sensing kinases AMP-activated protein kinase (AMPK) and mammalian target of rapamycin (mTOR) in adipocyte differentiation. IUBMB Life $\mathbf{6 5}$ 572-583. (https://doi.org/10.1002/iub.1170)

Fetissov SO 2016 Role of the gut microbiota in host appetite control: bacterial growth to animal feeding behaviour. Nature Reviews Endocrinology 13 11-25. (https://doi.org/10.1038/nrendo.2016.150)

Frank A, Brown LM \& Clegg DJ 2014 The role of hypothalamic estrogen receptors in metabolic regulation. Frontiers in Neuroendocrinology 35 550-557. (https://doi.org/10.1016/j.yfrne.2014.05.002)

Gautron L, Elmquist J \& Williams K 2015 Neural control of energy balance: translating circuits to therapies. Cell 161 133-145. (https:// doi.org/10.1016/j.cell.2015.02.023)

Guo J, Shu H, Wang L, Xu J, Niu X \& Zhang L 2017 SIRT1-dependent AMPK pathway in the protection of estrogen against ischemic brain injury. CNS Neuroscience and Therapeutics 23 360-369. (https://doi. org/10.1111/cns.12686)
Gyengesi E, Liu ZW, D'Agostino G, Gan G, Horvath TL, Gao XB \& Diano S 2010 Corticosterone regulates synaptic input organization of POMC and NPY/AgRP neurons in adult mice. Endocrinology 151 5395-5402. (https://doi.org/10.1210/en.2010-0681)

Hardie DG, Scott JW, Pan DA \& Hudson ER 2003 Management of cellular energy by the AMP-activated protein kinase system. FEBS Letters 546 113-120. (https://doi.org/10.1016/S00145793(03)00560-X)

Kocalis H, Turney M, Printz R, Laryea G, Muglia L, Davies S, Stanwood G, McGuinness O \& Niswender K 2012 Neuron-specific deletion of peroxisome proliferator-activated receptor delta (PPAR $\delta$ ) in mice leads to increased susceptibility to diet-induced obesity. PLoS ONE 7 e42981. (https://doi.org/10.1371/journal.pone.0042981)

Lachize S, Apostolakis EM, van der Laan S, Tijssen AM, Xu J, de Kloet ER \& Meijer OC 2009 Steroid receptor coactivator-1 is necessary for regulation of corticotropin-releasing hormone by chronic stress and glucocorticoids. PNAS 106 8038-8042. (https://doi.org/10.1073/ pnas.0812062106)

Lee SK, Kim HJ, Na SY, Kim TS, Choi HS, Im SY \& Lee JW 1998 Steroid receptor coactivator-1 coactivates activating protein-1-mediated transactivations through interaction with the c-Jun and c-Fos subunits. Journal of Biological Chemistry 273 16651-16654. (https:// doi.org/10.1074/jbc.273.27.16651)

Louet JF, Chopra AR, Sagen JV, An J, York B, Tannour-Louet M, Saha PK, Stevens RD, Wenner BR, Ilkayeva OR, et al. 2010 The coactivator SRC-1 is an essential coordinator of hepatic glucose production. Cell Metabolism 12 606-618. (https://doi.org/10.1016/j.cmet.2010.11.009)

Meijer OC, Steenbergen PJ \& De Kloet ER 2000 Differential expression and regional distribution of steroid receptor coactivators SRC-1 and SRC-2 in brain and pituitary. Endocrinology 141 2192-2199. (https:// doi.org/10.1210/endo.141.6.7489)

Minokoshi Y, Alquier T, Furukawa N, Kim YB, Lee A, Xue B, Mu J, Foufelle F, Ferre P, Birnbaum MJ, et al. 2004 AMP-kinase regulates food intake by responding to hormonal and nutrient signals in the hypothalamus. Nature $\mathbf{4 2 8}$ 569-574. (https://doi.org/10.1038/ nature02440)

Morford J, Wu S \& Mauvais-Jarvis F 2017 The impact of androgen actions in neurons on metabolic health and disease. Molecular and Cellular Endocrinology 465 92-102. (https://doi.org/10.1016/j. mce.2017.09.001)

Morton GJ, Cummings DE, Baskin DG, Barsh GS \& Schwartz MW 2006 Central nervous system control of food intake and body weight. Nature 443 289-295. (https://doi.org/10.1038/nature05026)

Na SY, Lee SK, Han SJ, Choi HS, Im SY \& Lee JW 1998 Steroid receptor coactivator-1 interacts with the p50 subunit and coactivates nuclear factor kappaB-mediated transactivations. Journal of Biological Chemistry 273 10831-10834. (https://doi.org/10.1074/ jbc.273.18.10831)

Nishihara E, Yoshida-Komiya H, Chan CS, Liao L, Davis RL, O'Malley BW \& Xu J 2003 SRC-1 null mice exhibit moderate motor dysfunction and delayed development of cerebellar Purkinje cells. Journal of Neuroscience 23 213-222. (https://doi.org/10.1523/ JNEUROSCI.23-01-00213.2003)

Onate SA, Tsai SY, Tsai MJ \& O'Malley BW 1995 Sequence and characterization of a coactivator for the steroid hormone receptor superfamily. Science 270 1354-1357. (https://doi.org/10.1126/ science.270.5240.1354)

Picard F, Géhin M, Annicotte JS, Rocchi S, Champy MF, O'Malley BW, Chambon P \& Auwerx J 2002 SRC-1 and TIF2 control energy balance between white and brown adipose tissues. Cell 111 931-941. (https://doi.org/10.1016/S0092-8674(02)01169-8)

Ryan KK Li B Grayson BE 2011 A Role for Central Nervous System PPAR- $\gamma$ in the Regulation of Energy Balance. Nature Medicine 17 623-626. (https://doi.org/10.1038/nm.2349)

Sefton C, Harno E, Davies A, Small H, Allen T, Wray J, Lawrence C, Coll A \& White A 2016 Elevated hypothalamic glucocorticoid levels 
are associated with obesity and hyperphagia in male mice. Endocrinology 157 4257-4265. (https://doi.org/10.1210/en.2016-1571)

Shrivastav A, Bruce M, Jaksic D, Bader T, Seekallu S, Penner C, Nugent Z, Watson P \& Murphy L 2014 The mechanistic target for rapamycin pathway is related to the phosphorylation score for estrogen receptor- $\alpha$ in human breast tumors in vivo. Breast Cancer Research 16 R49. (https://doi.org/10.1186/bcr3660)

Stashi E, York B \& O'Malley BW 2014 Steroid receptor coactivators: servants and masters for control of systems metabolism. Trends in Endocrinology and Metabolism 25 337-347. (https://doi.org/10.1016/j. tem.2014.05.004)

Wade GN \& Zucker I 1970 Modulation of food intake and locomotor activity in female rats by diencephalic hormone implants. Journal of Comparative and Physiological Psychology 72 328-336. (https://doi. org $/ 10.1037 / \mathrm{h} 0029461)$

Winnay JN, Xu J, O'Malley BW \& Hammer GD 2006 Steroid receptor coactivator-1-deficient mice exhibit altered hypothalamic-pituitary- adrenal axis function. Endocrinology 147 1322-1332. (https://doi. org/10.1210/en.2005-0751)

York B \& O'Malley BW 2010 Steroid receptor coactivator (SRC) family: masters of systems biology. Journal of Biological Chemistry $\mathbf{2 8 5}$ 38743-38750. (https://doi.org/10.1074/jbc.R110.193367)

Zhang D, Guo Q, Bian C, Zhang J, Lin S \& Su B 2011 Alterations of steroid receptor coactivator-1 (SRC-1) immunoreactivities in specific brain regions of young and middle-aged female Sprague-Dawley rats. Brain Research 1382 88-97. (https://doi.org/10.1016/j.brainres.2011.01.024)

Zhu Y, Qi C, Calandra C, Rao MS \& Reddy JK 1996 Cloning and identification of mouse steroid receptor coactivator-1 (mSRC-1), as a coactivator of peroxisome proliferator-activated receptor gamma. Gene Expression 6 185-195.

Zhu L, Yang Y, Xu P, Zou F, Yan X, Liao L, Xu J, O'Malley B \& Xu Y 2013 Steroid receptor coactivator-1 mediates estrogenic actions to prevent body weight gain in female mice. Endocrinology $\mathbf{1 5 4}$ 150-158. (https://doi.org/10.1210/en.2012-2007)

Received in final form 8 October 2018

Accepted 25 October 2018

Accepted Preprint published online 29 October 2018
(C) 2019 Society for Endocrinology Published by Bioscientifica Ltd. Printed in Great Britain 